\title{
Neural correlates of visual memory in Diffuse Axonal Injury patients
}

Juliane Lauer $^{1 *}$, Laura Moreno-López ${ }^{1 *}$, Anne Manktelow ${ }^{1}$, Ellen L. Carroll ${ }^{1}$, Joanne G. Outtrim ${ }^{1}$, Jonathan P. Coles ${ }^{1}$, Virginia F. Newcombe ${ }^{1,3}$, Barbara J. Sahakian ${ }^{2,3}$, David K. Menon ${ }^{1,3}$ and Emmanuel A. Stamatakis ${ }^{1,3}$

${ }^{1}$ Division of Anaesthesia, University of Cambridge, Cambridge, UK

${ }^{2}$ Department of Psychiatry and MRC/Wellcome Trust Behavioural and Clinical Neuroscience Institute, University of Cambridge, Cambridge, UK

${ }^{3}$ Wolfson Brain Imaging Centre, Department of Clinical Neurosciences, University of Cambridge, Cambridge Biomedical Campus, Cambridge, UK

* J. Lauer and L. Moreno-López contributed equally to this article.

\section{Correspondence:}

Laura Moreno-Lopez

Division of Anaesthesia

Box 93 Addenbrooke's Hospital, Hills Road,

Cambridge, CB2 0QQ, UK

+44(0)1223217892

$\underline{\operatorname{lm618@cam.ac.uk}}$ 


\begin{abstract}
In this study, 39 patients spanning the full range of traumatic brain injury (TBI) severity and a group of 53 matched healthy controls (HC) were evaluated on CANTAB tests of visual-spatial short-term recognition memory and learning. ANCOVAs and Cohen's d estimations of effect sizes were used to test between-group differences in the measures of memory function. Partial correlations and multiple regression analyses were used to examine relationships between cognitive variables and MRI volumetric findings. Patients with TBI performed significantly worse than controls in all the tasks assessed. Performance in the tests was associated with reductions in grey and white matter volume of several cortical and subcortical structures as well as with CSF space enlargement in accordance with previous studies of memory in patients with TBI and cognitive models that suggest memory problems involve the alteration of multiple systems. Our results propose that compromised visual memory in patients with TBI is related to a distributed pattern of volume loss in regions mediating memory and attentional processing.
\end{abstract}

\title{
Keywords
}

Traumatic Brain Injury, Visual Memory, Magnetic Resonance Imaging 


\section{Introduction}

Traumatic brain injury (TBI) is associated with a wide variety of cognitive and emotional deficits that can persist several years after injury and have an effect on psychosocial recovery. Common cognitive problems associated with mild, moderate and severe TBI include difficulties with memory, attention, information processing and executive function [1,2]. Memory is one of the functions most frequently impaired following TBI $[3,4]$. Such problems, noted by patients and family members [5], significantly influence an individual's rehabilitation and have been associated with poorer functional outcome (e.g. employment status, emotional functioning and overall quality of life) [6].

It is currently accepted that memory is not a unitary system but a set of systems with different neuroanatomical substrates [7]. Memory has been classified into different subtypes depending on its persistence (short or long-term memory), the contents of its stored material (episodic or semantic), and the presence or not of consciousness during learning and memory (declarative or nondeclarative). Deficits in declarative memory - the capacity for conscious recollection of facts and events - are a common consequence of head trauma that is disproportionately suffered in comparison with other types of memory [8]. Neuroanatomically, this kind of memory has been associated with the integrity of the prefrontal cortex and medial temporal lobes [9-11] the fusiform [12,13], the parietal [14,15] and the premotor cortex $[16,17]$. These regions are thought to be involved in the encoding and transformation of sensory input into internal representations (inferior frontal and fusiform gyrus) [18], storage of the internal representations into memory (medial temporal lobe and hippocampal formation) [19,20] and attentional processing during encoding (parietal and premotor cortex) [21]. In patients with TBI, reductions in the volume of grey and white matter of the temporal, frontal, parietal and occipital lobes, as well as in the cingulate gyrus, the cerebellum and subcortical structures such as the basal ganglia and the thalamus have been associated with impaired memory functions [22-29]. Research 
into the neurobiological correlates of memory in TBI patients can increase our understanding of the underlying changes that occur in the brain following injury and identify potential targets for pharmacological modulations of disrupted neurotransmitter systems. However, many of the studies conducted so far are compromised by small sample sizes, variability in imaging methods or tests used and the use of samples with a mixture of focal and diffuse brain injuries [30]. Furthermore, previous work has typically focused on a limited number of regions of interest [31] and since most of the functions commonly impaired depend on the coherent activity of widely distributed networks [32,33], such approaches limit the scope for uncovering a complete picture for the structural causes of the pathology studied.

This study investigated the neural substrates of visual recognition memory in a sample of 39 patients with TBI. This study complements and extends previous studies by performing volumetric comparisons on a variety of resolution levels, from whole brain to voxel-based level analysis. Moreover, only patients with diffusion axonal injury (DAI), as opposed to focal lesions, were included, enabling us to study TBI-related memory impairment as a consequence of axonal damage and therefore compromised connectivity. We hypothesised that patients with decreased grey and white matter volume in the temporal, frontal, parietal and occipital lobes as well as in subcortical areas and the cerebellum would perform poorly on the tests of memory function analysed.

\section{Materials and Methods}

\subsection{Participants}

Thirty-nine patients with TBI were included in this study. Patients were referred from the Addenbrooke's Neurosciences Critical Care Unit Follow-Up Clinic, the Addenbrooke's Traumatic Brain Injury Clinic and the Royal London Hospital Intensive Care Unit. Only patients with DAI as confirmed by the Marshall Score [34]- were included in this study. They were at least 6 months 
post TBI (mean $14.09 \pm 9.17$ months) and were not receiving any acute hospital interventions. They sustained mild to severe TBI -as measured by the Glasgow Coma Scale (GCS) [35]. A group of 53 healthy controls (HC) recruited for studies running in parallel to ours, was used as a control group for comparison with the TBI patients in the neuropsychological tasks used. HC were recruited via advertisements in Addenbrooke's Hospital and in the local Cambridge area and were paid for their participation. Both TBI patients and HC were screened by exclusion criteria including National Adult Reading Test <70, Mini Mental State Exam <23, left-handedness, history of psychiatric or neurologic disorders, taking medication that may affect their physical or cognitive performance, history of drug or alcohol abuse and pregnancy or breast feeding. Additionally, patients were excluded if they had a physical disability that could prevent them from completing the tasks either in the screening or scanning stages. This study was approved by the local Research Ethics Committee. All participants gave written informed consent before participation according to the 1991 Declaration of Helsinki.

\subsection{Neuropsychological assessment}

Participants completed a series of computerised tasks, taken from the Cambridge Neuropsychological Test Automated Battery (CANTAB) (http://www.cantab.com). Specifically, the Patter recognition memory (PRM), the Spatial recognition memory (SRM) and the Paired associates learning (PAL) were used.

\subsubsection{Pattern Recognition Memory}

The PRM task employs a two-choice forced discrimination paradigm. One at a time, 12 different visual patterns, which are designed in a way that makes it difficult to assign verbal labels to them, appear on a computer screen. Subsequently, the participant is asked to discriminate between previously presented and novel patterns. This procedure is repeated with 12 new patterns. The test is a good indicator of overall visual short-term recognition memory, which is impaired in disorders 
such as mild to moderate Alzheimer's disease [36,37]. Here, TBI patients' performance was evaluated based on the number of correct trials, expressed as a percentage (i.e. percentage of correct trials). Higher percentage indicates better performance.

\subsubsection{Spatial Recognition Memory}

The second task designed to test participants' visual memory was the SRM. This task is based on a two-choice discrimination paradigm: a white square appears sequentially at different locations on the screen. Following this, in the recognition phase, the participant is presented with a series of pairs of squares, with one of those being in a previously seen position. The other square is in a novel position on the screen. This test is repeated three more times, each time with new locations. The outcome measure chosen for this task was the participants' speed (mean latency for correct responses). Latency is scored in milliseconds with lower latency indicating better performance. SRM has previously shown higher latency scores in TBI compared to healthy controls [38].

\subsubsection{Paired Associates Learning}

PAL combines the different visual memory components evaluated by the PRM and SRM tasks. It assesses visual memory and new learning and is sensitive to mild cognitive impairment and Alzheimer's disease [23,39]. Since TBI has been associated with increased risk of these disorders [40-42], the combination of neuroimaging and the PAL may provide insights into one of the possible mechanisms by which risk for neurodegenerative disorders is increased following TBI.

The PAL involves boxes displayed on the screen opening in a random order, with one or more of them containing a pattern. Subsequently, the patterns appear in the middle of the screen, one at a time. The participant is asked to touch the box in which the pattern was initially presented. Should the participant make a mistake, the patterns are presented again as a reminder of the original locations. Task difficulty increases with the number of patterns presented (up to eight). The analysis of PAL focused on the number of errors made at the 6-pattern stage. The 6- 
pattern condition has been found to be reliable in distinguishing between individuals with memory impairments and healthy controls, as well as differentiating between various neurodegenerative conditions [e.g. 36,37,43]. Lower number of errors indicates better performance.

\subsection{MRI acquisition and preprocessing}

The imaging session started with a localiser followed by a high resolution T1-weighted, magnetization-prepared 180 degrees radio-frequency pulses, rapid gradient-echo (MPRAGE) structural scan $\left(\mathrm{TR}=2300 \mathrm{~ms}, \mathrm{TE}=2.98 \mathrm{~ms}, \mathrm{TA}=9.14 \mathrm{~min}\right.$, flip angle $=9^{\circ}, \mathrm{FOV}=256 \mathrm{~mm}$, voxel size $=1.0 \times 1.0 \times 1.0 \mathrm{~mm}$, slices $=176)$, acquired on a 3T Siemens Trio scanner $($ Siemens AG, Erlangen, Germany) at the Wolfson Brain Imaging Centre (WBIC), University of Cambridge.

The images were preprocessed and analysed using SPM8 (http://www.fil.ion.ucl.ac.uk/spm/software/spm8/). Initially, the T1 images were segmented into grey matter, white matter and cerebrospinal fluid (CSF) using the VBM8-toolbox (http://dbm.neuro.uni-jena.de/vbm/download/). The images were entered into the DARTEL framework and high resolution study-specific grey matter, white matter and CSF templates were created and used as tissue probability maps in an additional normalisation and segmentation of the original T1 scans. Segmented images were subjected to Jacobian modulation to sensitise the analyses to true volumetric differences between patients. Although this cohort suffered diffuse injuries and therefore did not present with anatomical distortions we used study specific templates to optimise spatial normalisation. We visually inspected the images following spatial normalisation to ascertain the algorithm transformed the images to standard space. Finally, the images were smoothed with a 3-D Gaussian filter of $8 \mathrm{~mm}$ full width at half maximum (FWHM).

Individual grey matter, white matter and CSF volumes were calculated for the whole brain, the left and right hemispheres (separated into brainstem, cerebellum and cerebrum), the midbrain (including the pons, medulla and midbrain) and separate lobes (fronto-temporal space, the frontal, 
limbic, occipital, parietal and temporal lobe, as well as the medulla, pons, sublobar region and anterior and posterior cerebellum). Additionally, a volumetric analysis was performed using the areas defined by Automated Anatomical Labeling (AAL) atlas [44]. Finally, a more detailed voxel-level analysis was performed [45]. Because there can be differences in results when ROI vs. voxel-based approaches are employed, the use of different approaches in the same dataset could help to establish structural differences that converge across analysis methods, as well as, the level of resolution sufficient to detect behavioural/structural associations (see Supplementary Figure 1 for an illustration of the different levels of resolution used).

\subsection{Analyses}

\subsubsection{Behavioural analysis}

Behavioural data were analysed with the SPSS 21 (Chicago, IL, USA). Independent-sample t-test and chi-square tests were used to compare the groups on relevant socio-demographic variables. ANCOVAs and Cohen's d estimations of effect sizes were used to test between-group differences in the measures of memory functioning. Due to the large variance in the age of the participants and previously reported age effects in brain morphometry $[28,46]$ and neuropsychological performance [47-49], age at assessment was used as a covariate in our analyses. Significance threshold was set at $\mathrm{p}<0.05$.

\subsubsection{Imaging analysis}

Analyses were conducted using the SPSS 21 in the cases of whole brain, hemisphere, lobe and gyri volume comparisons and SPM8 for the voxel-based correlations between regional grey matter, white matter and CSF and the scores of the different memory scales. Partial correlations and multiple regression analyses were used to examine possible relationships between cognitive variables and MRI volumetric findings. Age was used as a covariate in all the analyses. Significance threshold was set at $\mathrm{p}<0.05$ in the first set of analysis (SPSS) and at a $\mathrm{p}$ value of 
$<0.001$ uncorrected for voxels and $<0.05$ at cluster-level with correction for family-wise error (FWE) in the second one.

\section{Results}

\subsection{Behavioural analyses}

The groups were statistically matched in terms of gender $\left(x^{2}=0.694, p=0.405\right)$, age $\left(t_{89}=-0.425\right.$, $\mathrm{p}=0.672)$, and premorbid IQ ( $\left.\mathrm{t}_{89}=-1.526, \mathrm{p}=0.132\right)$, as estimated by the National Adult Reading Test (NART). Demographic and characteristics of the samples are summarized in Table 1. The results of the analyses showed that patients with TBI had significantly poorer performance than HC on all neuropsychological tests administered (Table 2). Calculation of effect sizes showed large effect sizes $(d>0.8)$ for the PAL and medium effect sizes $(d>0.5)$ for the PRM and the SRM (Table 2).

Please insert Tables 1 and 2 here

\subsection{Imaging analyses}

\subsection{Pattern Recognition Memory}

Amounts of grey and white matter in the temporal lobe were found to be significantly correlated with percent of correct responses. Patients with more intact tissue outperformed patients with lower amounts of grey $(\mathrm{r}=0.36, \mathrm{p}<0.05)$ and white matter $(\mathrm{r}=0.38, \mathrm{p}<0.05)$. Positive correlations between tissue volume and performance were also found at the gyral level (see Table 3). Specifically, we found significant positive correlations between the percentage of correct trials and gray matter volume in the temporal and occipital lobes, the angular gyrus and the putamen and white matter volume in the hippocampus, the temporal and frontal lobes, the angular gyrus and the cerebellum. Furthermore, a negative correlation between CSF volume in the right hippocampus and PRM accuracy was found, which is in line with the positive association found between 
performance and amount of intact white matter in this region. Finally, we found a significant positive correlation between accuracy and CSF volume in the areas 4 and 5 of the vermis. The analysis at the voxel-levels did not show significant correlations at the threshold level used.

\section{Please insert Table 3 here}

\subsection{Spatial Recognition Memory}

Lower resolution level analyses did not yield any significant results. Analyses on gyral-level were more informative (Table 4). Patients with higher amounts of grey matter in the hippocampus and parts of the vermis responded faster. The same was true for more intact white matter volume in a number of bilateral frontal structures. Additionally, more intact white matter in the left cuneus was positively related to mean latency. Finally, a negative correlation between CSF volume in the right cerebellum (area 10) and speed was found, indicating a correlation between greater grey or white matter atrophy and slower responses.

\section{Please insert Table 4 here}

\subsection{Paired Associates Learning}

Patients with more intact grey matter in the frontal $(r=-0.43, \mathrm{p}<0.01)$, temporal $(r=-0.44, \mathrm{p}<0.01)$, parietal $(\mathrm{r}=-0.46, \mathrm{p}<0.01)$ and limbic lobes $(\mathrm{r}=-0.34, \mathrm{p}<0.05)$, as well as sublobar regions $(\mathrm{r}=-0.46$, $\mathrm{p}<0.01)$ were found to make significantly fewer errors. The association between sublobar tissue volume and errors made was also found in the white matter analysis $(r=-0.34, p<0.05)$. Also, higher CSF volume in the parietal $(r=-0.42, p<0.05)$ and occipital lobe $(r=-0.40, p<0.05)$, and thus potentially lower grey or white matter amounts, were linked to poorer performance.

The importance of intact tissue for successful PAL performance emerged again when analysing the data on a gyral level (Table 5). Lower grey and white matter volume in the temporal and frontal lobes, as well as in the hippocampus, supplementary motor area and basal ganglia were 
associated with impaired performance on this task. Similarly, patients with higher amounts of grey matter in the parietal and occipital cortex, rolandic operculum, cingulum, precentral, fusiform and angular gyrus, in addition to the white matter volume of the thalamus and the cerebellum made fewer errors on this task. In contrast, there was a significant positive correlation between more intact white matter in the middle temporal gyrus (temporal pole) and the number of errors made. The correlation results of PAL performance and CSF volume on gyral level are consistent with the grey and white matter findings at this level of analysis. In this sense, less CSF, and therefore probably higher amounts of brain tissue, was associated with better performance. Significant results for this relationship were found in parts of the hippocampus, temporal, frontal and occipital lobes and the cerebellum.

The voxel based analysis revealed significant negative correlations between the number of errors and grey matter volume in the medial part of the superior frontal gyrus, the precentral gyrus, and the precuneus. Similarly, more intact white matter in the right sagittal stratum and near the inferior and middle temporal gyri, with the cluster extending to the fusiform gyrus, was associated with better performance. The importance of intact brain tissue for good PAL performance was echoed in the CSF VBM results. Increased CSF near temporal, frontal and occipital regions was linked with poorer performance (Fig. 1).

Please insert Table 5 and Fig. 1 here

\section{Discussion}

Consistent with a body of literature on the presence of memory deficits in patients with TBI $[4,50]$, our patients showed significant impairments in the measures of visual memory analysed. When the neural correlates of memory function were analyzed, memory performance was associated with reductions in the volume of grey and white matter of several cortical and subcortical structures as well as with ventricular enlargement. This finding is in accordance with 
previous studies of memory in patients with TBI and theoretical models proposing that memory problems in patients with TBI involve the alteration of multiple systems [25,32]. Along these lines, it has been suggested that memory deficits in this population may result from difficulties in the application of active or effortful strategies in learning or retrieval processes [4,51]. Moreover, the involvement of numerous regions in memory performance in this population might indicate the use of compensatory mechanisms to optimize performance [52,53]. Our data therefore suggest that memory impairments persist following TBI highlighting the chronic nature of this condition [5457].

The first task we used to assess patients' visual memory abilities was the PRM test. The results of our study implicate the hippocampus and the temporal lobe in PRM performance. The hippocampus and the temporal lobe have been consistently associated with memory functioning. Specifically, evidence indicates that both structures are involved in the storage of internal representations into memory $[19,20]$.

Furthermore, both structures have been associated with visual recognition memory in previous studies of healthy individuals and patients with temporal lobe damage [47,49-51,53-60]. We also found a positive correlation between the volume of white matter of the inferior frontal gyrus and performance in this task. This finding is in line with the role of this region in the encoding and recognition of visual and verbal information [61-65]. The memory deficits we found in the patients with TBI and their association with structures of the temporo-parietal network, are comparable to the abnormalities reported in patients with Alzheimer's disease and mild cognitive impairment [e.g. 23,66]. This finding provides some insight into the increased dementia risk documented in TBI patients $[67,68]$. Future research should explore the sensitivity and specificity of neuropsychological and neuroimaging biomarkers in this population and provide differential treatment pathways and rehabilitation strategies. 
Our findings also suggest that intact tissue in the left angular gyrus is critical for successful PRM performance. This result overlaps with previous findings demonstrating the importance of this structure in learning and memory processes [69]. For example, it has been postulated that the angular gyri may directly support episodic retrieval through their anatomical and functional connections with the medial temporal lobe [70-73]. The analyses at the gyral-level also implicated the occipital cortex and the cerebellum in this task. Both structures have showed increased activation during a visual memory task [74]. The involvement of the middle occipital gyrus might reflect its known role in visual processing and object recognition [75].

The cerebellum, on the other hand, has been found to be involved in memory function, among other cognitive domains [76-78]. Further evidence for this association was found in a study of twenty-three children with severe traumatic brain injury [22]. According to the authors, cerebellar lesions can lead to reduced performance in tasks that require sustained attention such as tasks of visual recognition memory $[22,79]$. We also found a link between lower grey matter in the putamen and PRM performance. This association coincides with recent studies showing the role of the basal ganglia in feedback-based learning [38].

Investigation of the neural correlates of the SRM suggested that more intact grey matter volume in the hippocampus and cerebellar areas support faster correct responses on this task, consistent with the studies that show that TBI patients with compromised tissue in these areas responded significantly slower when compared to $\mathrm{HC}$ [38]. White matter volume in the inferior and middle frontal gyri was associated with better performance, in accordance with the role of these structures in the encoding and recognition of visual and verbal information [61-65]. We also found a positive correlation between the volume of white matter in the cuneus (a region of the default mode network) and the participants' speed in line with studies that show that disrupted structural grey and white-matter integrity in the default mode network. These findings suggest a potential mechanism for the structural changes found in this population [80,81]. 
Finally, the PAL results showed that performance in this task relies on a wide range of structures, including the frontal, temporal, parietal and occipital lobes, as well as medial (i.e. cingulate, precentral gyrus and supplementary motor area) and subcortical regions (i.e. basal ganglia, thalamus and pallidum), the default mode network (i.e. precuneus and angular gyrus) and the cerebellum. In addition to the role of the regions found associated with memory performance in the PRM and SRM tests, the number of errors in the PAL was associated with grey matter volume in the parietal gyrus. The role of the parietal cortex in memory function has been emphasized in previous studies. For example, greater activations in specific parietal regions have been associated with better episodic memory retrieval, recognition of old items, as well as familiarity of events [82-84]. Alterations in the parietal cortex are known to cause attentional dysfunction during processes that support memory retrieval, searching, monitoring and verification [85]. Reduced grey matter volume in medial structures such as the cingulate, precentral gyrus and the supplementary motor area were associated with a higher number of errors. These regions have been associated with attentional processing, an important element of memory encoding $[16,17,21]$. We also found a negative correlation between the volume of white matter of several subcortical structures and the performance in this task, in line with recent studies showing the involvement of subcortical structures in memory functioning [86-88]. Finally, the volume of grey matter of the rolandic operculum and the fusiform gyrus were associated with PAL performance in accordance with the role of these structures in somatosensory processing $[12,13,18,89]$.

This study investigated the neural substrates of visual recognition memory in a sample of 39 patients with TBI. Strengths of this study include the use of an optimized VBM protocol, as well as the use of several resolution levels in the analyses. We found the voxel-level analyses were not very informative, a result that could reflect the differences in statistical power between voxelbased and ROI analyses [90] but also the heterogeneity inherent in TBI. Limitations of this study 
include the heterogeneity of our sample and the exploratory nature of the analyses. In this sense, our study included individuals with a wide range of injury severities and time since injury. Nevertheless, although the sample studied was heterogeneous, the patients were characterized as having DAI and age at assessment was included as covariate. 


\section{Declaration of interest}

The study was funded by the Evelyn Trust- grant number 06/20. L.M.L. was funded by the Division of Anaesthesia of the University of Cambridge. A.M. is supported by CLAHRC-East of England awards. E.L.C., J.G.O., J.P.C. and D.K.M. are funded by the Neuroscience Theme of the NIHR Cambridge Biomedical Research Centre and NIHR and by Framework Program 7 funding from the European Commission (CENTER-TBI). B.J.S. consults for Cambridge Cognition, Otsuka, Servier and Lundbeck. She holds a grant from Janssen/J\&J and has share options in Cambridge Cognition. D.K.M also received lecture and consultancy fees and support for research from Glaxo SmithKline, Solvay and Linde. E.A.S. is funded by the Stephen Erskine Fellowship, Queens' College, Cambridge, UK. V.F.J.N. is supported by a Health Foundation/Academy of Medical Sciences Clinician Scientist Fellowship. 


\section{References}

[1]. McAllister TW. Neurobiological consequences of traumatic brain injury. Dialogues Clin Neurosci 2011; 13: 287-300.

[2]. Schretlen DJ, Shapiro AM. A quantitative review of the effects of traumatic brain injury on cognitive functioning. Int Rev Psychiatry 2003; 15: 341-9.

[3]. Fork M, Bartels C, Ebert AD, Grubich C, Synowitz H, Wallesch CW. Neuropsychological sequelae of diffuse traumatic brain injury. Brain Inj 2005; 19: 101-8.

[4]. Vakil E. The effect of moderate to severe traumatic brain injury (TBI) on different aspects of memory: a selective review. J Clin Exp Neuropsychol 2005; 27: 977-1021.

[5]. Corrigan JD, Whiteneck G, Mellick D. Perceived needs following traumatic brain injury. J Head Trauma Rehabil 2004; 19: 205-16.

[6]. Wood RL, Rutterford NA. Demographic and cognitive predictors of long-term psychosocial outcome following traumatic brain injury. J Int Neuropsychol Soc 2006; 12: 350-8.

[7]. Squire LR, Zola-Morgan S. The medial temporal lobe memory system. Science 1991; 253: $1380-6$.

[8]. Palacios EM, Fernandez-Espejo D, Junque C, Sanchez-Carrion R, Roig T, Tormos JM, et al. Diffusion tensor imaging differences relate to memory deficits in diffuse traumatic brain injury. BMC Neurol 2011; 11: 24.

[9]. Buckner RL, Wheeler ME, Sheridan MA. Encoding processes during retrieval tasks. J Cogn Neurosci 2001; 13: 406-15. 
[10]. Fernández G, Tendolkar I. Integrated brain activity in medial temporal and prefrontal areas predicts subsequent memory performance: human declarative memory formation at the system level. Brain Res Bull 2001; 55: 1-9.

[11]. Simons J, Spiers H. Prefrontal and medial temporal lobe interactions in long term memory. Nat Rev Neurosci 2003; 4: 637-48.

[12]. Dickerson BC, Miller SL, Greve DN, Dale AM, Albert MS, Schacter DL, et al. Prefrontalhippocampal-fusiform activity during encoding predicts intraindividual differences in free recall ability: an event-related functional-anatomic MRI study. Hippocampus 2007; 17: 1060-70.

[13]. Kim H, Cabeza R. Differential contributions of prefrontal, medial temporal, and sensoryperceptual regions to true and false memory formation. Cereb Cortex 2007; 17: 2143-50.

[14]. Sommer T, Rose M, Weiller C, Büchel C. Contributions of occipital, parietal and parahippocampal cortex to encoding of object-location associations. Neuropsychologia 2005; 43: $732-43$.

[15]. Uncapher MR, Rugg MD. Selecting for memory? The influence of selective attention on the mnemonic binding of contextual information. J Neurosci 2009; 29: 8270-9.

[16]. Kao YC, Davis ES, Gabrieli JD. Neural correlates of actual and predicted memory formation. Nat Neurosci 2005; 8: 1776-83.

[17]. Morcom AM, Good CD, Frackowiak RS, Rugg MD. Age effects on the neural correlates of successful memory encoding. Brain 2003; 126: 213-29.

[18]. Paller KA, Wagner AD. Observing the transformation of experience into memory. Trends Cogn Sci 2002; 6: 93-102. 
[19]. Diana RA, Yonelinas AP, Ranganath C. Imaging recollection and familiarity in the medial temporal lobe: a three-component model. Trends Cogn Sci 2007; 11: 379-86.

[20]. Squire LR, Stark CE, Clark RE. The medial temporal lobe. Annu Rev Neurosci 2004; 27: 279-306.

[21]. Kim H. Neural activity that predicts subsequent memory and forgetting: a meta-analysis of 74 fMRI studies. Neuroimage 2011; 54: 2446-61.

[22]. Braga LW, Souza LN, Najjar YJ, Dellatolas G. Magnetic resonance imaging (MRI) findings and neuropsychological sequelae in children after severe traumatic brain injury: the role of cerebellar lesion. J Child Neurol 2007; 22: 1084-9.

[23]. De Rover M, Pironti VA, McCabe JA, Acosta-Cabronero J, Arana FS, Morein-Zamir S, et al. Hippocampal dysfunction in patients with mild cognitive impairment: a functional neuroimaging study of a visuospatial paired associates learning task. Neuropsychologia 2011; 49: 2060-70.

[24]. Himanen L, Portin R, Isoniemi H, Helenius H, Kurki T, Tenovuo O. Cognitive functions in relation to MRI findings 30 years after traumatic brain injury. Brain Inj 2005; 19: 93-100.

[25]. Levine B, Kovacevic N, Nica EI, Schwartz ML, Gao F, Black SE. Quantified MRI and cognition in TBI with diffuse and focal damage. Neuroimage Clin 2013; 2: 534-41.

[26]. Palacios EM, Sala-Llonch R, Junque C, Fernandez-Espejo D, Roig T, Tormos JM, et al. Long-term declarative memory deficits in diffuse TBI: correlations with cortical thickness, white matter integrity and hippocampal volume. Cortex 2013; 49: 646-57.

[27]. Serra-Grabulosa JM, Junqué C, Verger K, Salgado-Pineda P, Mañeru C, Mercader JM. Cerebral correlates of declarative memory dysfunctions in early traumatic brain injury. $\mathrm{J}$ Neurol Neurosurg Psychiatry 2005; 76: 129-31. 
[28]. Spitz G, Bigler ED, Abildskov T, Maller JJ, O'Sullivan R, Ponsford JL. Regional cortical volume and cognitive functioning following traumatic brain injury. Brain $\operatorname{Cogn} 2013 ; 83$ : 34-44. [29]. Vannorsdall TD, Cascella NG, Rao V, Pearlson GD, Gordon B, Schretlen DJ. A morphometric analysis of neuroanatomic abnormalities in traumatic brain injury. $\mathbf{J}$ Neuropsychiatry Clin Neurosci 2010; 22: 173-81.

[30]. Levine B, Fujiwara E, O'Connor C. In vivo characterization of traumatic brain injury neuropathology with structural and functional neuroimaging. J Neurotrauma 2006; 23: 1396-411.

[31]. van der Naalt J, Hew JM, van Zomeren AH, Sluiter WJ, Minderhoud JM. Computed tomography and magnetic resonance imaging in mild to moderate head injury: early and late imaging related to outcome. Ann Neurol 1999; 46: 70-8.

[32]. Jeong W, Chung CK, Kim JS. Episodic memory in aspects of large-scale brain networks. Front Hum Neurosci 2015; 9: 454.

[33]. Sharp DJ, Scott G, Leech R. Network dysfunction after traumatic brain injury. Nat Rev Neurol 2014; 10: 156-66.

[34]. Marshall LF, Marshall SB, Klauber MR, Clark MvB, Eisenberg HM, Jane JA, et al. A new classification of head injury based on computerized tomography. J Neurosurg 1991; 75: S14-S20.

[35]. Teasdale G, Jennett B. Assessment of coma and impaired consciousness: a practical scale. Lancet 1974; 2: 81-4.

[36]. Sahakian BJ, Morris RG, Evenden JL, Heald A, Levy R, Philpot M, et al. A comparative study of visuospatial memory and learning in Alzheimer-type dementia and Parkinson's disease. Brain 1988; 111: 695-718. 
[37]. Swainson R, Hodges JR, Galton CJ, Semple J, Michael A, Dunn BD, et al. Early detection and differential diagnosis of Alzheimer's disease and depression with neuropsychological tasks. Dement Geriatr Cogn Disord 2001; 12: 265-80.

[38]. Salmond CH, Chatfield DA, Menon DK, Pickard JD, Sahakian BJ. Cognitive sequelae of head injury: involvement of basal forebrain and associated structures. Brain 2005; 128: 189-200.

[39]. Barnett JH, Blackwell AD, Sahakian BJ, Robbins TW. The Paired Associates Learning (PAL) Test: 30 years of CANTAB Translational Neuroscience from laboratory to bedside in dementia research. In: Robbins TW, Sahakian BJ, editors. Current Topics in Behavioral Neurosciences: Translational Neuropsychopharmacology. Springer.

[40]. Bramlett HM, Dietrich WD. Long-term consequences of traumatic brain injury: current status of potential mechanisms of injury and neurological outcomes. J Neurotrauma 2015; 32: $1834-48$.

[41]. Gupta R, Sen N. Traumatic brain injury: a risk factor for neurodegenerative diseases. Rev Neurosci 2016; 27: 93-100.

[42]. Sundman MH, Hall EE, Chen NK. Examining the relationship between head trauma and neurodegenerative disease: a review of epidemiology, pathology and neuroimaging techniques. $\mathrm{J}$ Alzheimers Dis Parkinsonism 2014; 4.

[43]. Juncos-Rabadán O, Pereiro AX, Facal D, Reboredo A, Lojo-Seoane C. Do the Cambridge Neuropsychological Test Automated Battery episodic memory measures discriminate amnestic mild cognitive impairment? Int J Geriatr Psychiatry 2014; 29: 602-9.

[44]. Tzourio-Mazoyer N, Landeau B. Automated anatomical labeling of activations in SPM using a macroscopic anatomical parcellation of the MNI MRI single-subject brain. Neuroimage 2002; 15: 273-89. 
[45]. Ashburner J, Friston KJ. Voxel-based morphometry--the methods. Neuroimage 2000; 11: 805-21.

[46]. Smith CD, Chebrolu H, Wekstein DR, Schmitt FA, Markesbery WR. Age and gender effects on human brain anatomy: a voxel-based morphometric study in healthy elderly. Neurobiol Aging 2007; 28: 1075-87.

[47]. Blackwell AD, Barnett JH, Hayat S. The effect of age, sex and education on visuospatial paired associates learning ability: preliminary data from a British population study. Alzheimer's and Dementia 2010; 6: 485.

[48]. Rast P, Zimprich D. Individual differences and reliability of paired associates learning in younger and older adults. Psychol Aging 2009; 24: 1001-06.

[49]. Skolimowska J, Wesierska M, Lewandowska M, Szymaszek A, Szelag E. Divergent effects of age on performance in spatial associative learning and real idiothetic memory in humans. Behav Brain Res 2011; 218: 87-93.

[50]. Azouvi P, Vallat-Azouvi C, Belmont A. Cognitive deficits after traumatic coma. Prog Brain Res 2009; 177: 89-110.

[51]. Arenth PM, Russell KC, Scanlon JM, Kessler LJ, Ricker JH. Encoding and recognition after traumatic brain injury: neuropsychological and functional magnetic resonance imaging findings. $\mathrm{J}$ Clin Exp Neuropsychol 2012; 34: 333-44.

[52]. Gillis MM, Hampstead BM. A two-part preliminary investigation of encoding-related activation changes after moderate to severe traumatic brain injury: hyperactivation, repetition suppression, and the role of the prefrontal cortex. Brain Imaging Behav 2015; 9: 801-20. 
[53]. Olsen A, Brunner JF, Indredavik Evensen KA, Finnanger TG, Vik A, Skandsen T, et al. Altered cognitive control activations after moderate-to-severe traumatic brain injury and their relationship to injury severity and everyday-life function. Cereb Cortex 2015; 25: 2170-80.

[54]. Dikmen SS, Machamer JE, Powell JM, Temkin NR. Outcome 3 to 5 years after moderate to severe traumatic brain injury. Arch Phys Med Rehabil 2003; 84: 1449-57.

[55]. Newcombe VF, Correia MM, Ledig C, Abate MG, Outtrim JG, Chatfield D, et al. Dynamic changes in white matter abnormalities correlate with late improvement and deterioration following TBI: a diffusion tensor imaging study. Neurorehabil Neural Repair 2015; 30: 49-62.

[56]. Salmond CH, Menon DK, Chatfield DA, Pickard JD, Sahakian BJ. Changes over time in cognitive and structural profiles of head injury survivors. Neuropsychologia 2006; 44: 1995-98.

[57]. Zec RF, Zellers D, Belman J, Miller J, Matthews J, Femeau-Belman D, et al. Long-term consequences of severe closed head injury on episodic memory. J Clin Exp Neuropsychol 2001; 23: $671-91$.

[58]. Eichenbaumm H, Yonelinas AP, Ranganath C. The medial temporal lobe and recognition memory. Annu Rev Neurosci 2007; 30: 123-52.

[59]. Manns J, Squire L. Impaired recognition memory on the Doors and People Test after damage limited to the hippocampal region. Hippocampus 1999; 499: 495-9.

[60]. Owen A, Sahakian B, Semple J, Polkey CE, Robbins T. Visuo-spatial short-term recognition memory and learning after temporal lobe excisions, frontal lobe excisions or amygdalohippocampectomy in man. Neuropsychologia 1995; 33: 1-24.

[61]. Cansino S, Maquet P, Dolan RJ, Rugg MD. Brain activity underlying encoding and retrieval of source memory. Cereb Cortex 2002; 12: 1048-56. 
[62]. Fletcher PC, Shallice T, Dolan RJ. The functional roles of prefrontal cortex in episodic memory. I. Encoding Brain 1998a; 121: 1239-48.

[63]. Fletcher PC, Shallice T, Frith CD, Frackowiak RS, Dolan RJ. The functional roles of prefrontal cortex in episodic memory. II. Retrieval Brain 1998b; 121: 1249-56.

[64]. Kirchhoff BA, Wagner AD, Maril A, Stern CE. Prefrontal-temporal circuitry for episodic encoding and subsequent memory. J Neurosci 2000; 20: 6173-80.

[65]. Spaniol J, Davidson PS, Kim AS, Han H, Moscovitch M, Grady CL. Event-related fMRI studies of episodic encoding and retrieval: meta-analyses using activation likelihood estimation. Neuropsychologia 2009; 47: 1765-79.

[66]. Galluzzi S, Geroldi C, Amicucci G, Bocchio-Chiavetto L, Bonetti M, Bonvicini C, et al. Supporting evidence for using biomarkers in the diagnosis of MCI due to AD. J Neurol 2013; 260: $640-50$.

[67]. Lye TC, Shores EA. Traumatic brain injury as a risk factor for Alzheimer's disease: a review. Neuropsychol Rev 2000; 10: 115-29.

[68]. Smith DH, Johnson VE, Stewart W. Chronic neuropathologies of single and repetitive TBI: substrates of dementia? Nat Rev Neurol 2013; 9: 211-21.

[69]. Sestieri C, Corbetta M, Romani GL, Shulman GL. Episodic memory retrieval, parietal cortex, and the default mode network: functional and topographic analyses. J Neurosci 2011; 31: $4407-20$.

[70]. Rushworth MF, Behrens TE, Johansen-Berg H. Connection patterns distinguish 3 regions of human parietal cortex. Cereb Cortex 2006; 16: 1418-30. 
[71]. Uddin LQ, Supekar K, Amin H, Rykhlevskaia E, Nguyen DA, Greicius MD, et al. Dissociable connectivity within human angular gyrus and intraparietal sulcus: evidence from functional and structural connectivity. Cereb Cortex 2010; 20: 2636-46.

[72]. Vilberg KL, Rugg MD. Memory retrieval and the parietal cortex: a review of evidence from a dual-process perspective. Neuropsychologia 2008; 46: 1787-99.

[73]. Vincent JL, Snyder AZ, Fox MD, Shannon BJ, Andrews JR, Raichle ME, et al. Coherent spontaneous activity identifies a hippocampal-parietal memory network. J Neurophysiol 2006; 96: $3517-31$.

[74]. Reber P, Stark C, Squire L. Contrasting cortical activity associated with category memory and recognition memory. Learn Mem 1998; 5: 420-8.

[75]. James TW, Humphrey GK, Gati JS, Servos P, Menon RS, Goodale MA. Haptic study of three-dimensional objects activates extrastriate visual areas. Neuropsychologia 2002; 40: 1706-14.

[76]. Buckner RL. The cerebellum and cognitive function: 25 years of insight from anatomy and neuroimaging. Neuron 2013; 80: 807-15.

[77]. Schmahmann JD. The role of the cerebellum in cognition and emotion: personal reflections since 1982 on the dysmetria of thought hypothesis, and its historical evolution from theory to therapy. Neuropsychol Rev 2010; 20: 236-60.

[78]. Weis S, Klaver P, Reul J, Elger CE, Fernández G. Temporal and cerebellar brain regions that support both declarative memory formation and retrieval. Cereb Cortex 2004; 14: 256-67.

[79]. Gottwald B, Mihajlovic Z, Wilde B, Mehdorn HM. Does the cerebellum contribute to specific aspects of attention? Neuropsychologia 2003; 41: 1452-60. 
[80]. Bonnelle V, Leech R, Kinnunen KM, Ham TE, Beckmann CF, De Boissezon X, et al. Default mode network connectivity predicts sustained attention deficits after traumatic brain injury. J Neurosci 2011; 31: 13442-51.

[81]. Moreno-López L, Manktelow A, Sahakian BJ, Menon DK, Stamatakis EA. Anything goes? Regulation of the neural processes underlying response inhibition in TBI patients. Eur Neuropsychopharmacol, in press.

[82]. Todd JJ, Marois R. Capacity limit of visual short-term memory in human posterior parietal cortex. Nature 2004; 428: 751-4.

[83]. Wagner AD, Shannon BJ, Kahn I, Buckner RL. Parietal lobe contributions to episodic memory retrieval. Trends Cogn Sci 2005; 9: 445-53.

[84]. Yonelinas AP, Otten LJ, Shaw KN, Rugg MD. Separating the brain regions involved in recollection and familiarity in recognition memory. J Neurosci 2005; 25: 3002-8.

[85]. Cabeza R, Ciaramelli E, Olson IR, Moscovitch M. The parietal cortex and episodic memory: an attentional account. Nat Rev Neurosci 2008; 9: 613-25.

[86]. Jankowski MM, Ronnqvist KC, Tsanov M, Vann SD, Wright NF, Erichsen JT, et al. The anterior thalamus provides a subcortical circuit supporting memory and spatial navigation. Front Syst Neurosci 2013; 7: 45.

[87]. McNab F, Klingberg T. Prefrontal cortex and basal ganglia control access to working memory. Nat Neurosci 2008; 11: 103-7.

[88]. Štillová K, Jurák P, Chládek J, Chrastina J, Halámek J, Bočková M, et al. The role of anterior nuclei of the thalamus: a subcortical gate in memory processing: an intracerebral recording study. PLoS One 2015; 10: e0140778. 
[89]. Eickhoff SB, Amunts K, Mohlberg H, Zilles K. The human parietal operculum. II. Stereotaxic maps and correlation with functional imaging results. Cereb Cortex 2006; 16: 268-79.

[90]. D'Mello AM, Crocetti D, Mostofsky SH, Stoodley CJ. Cerebellar gray matter and lobular volumes correlate with core autism symptoms. Neuroimage Clin 2015; 7: 631-9. 


\section{Tables}

Table 1. Demographic and clinical characteristics of the samples.

\begin{tabular}{|c|c|c|c|c|c|c|c|c|}
\hline & \multicolumn{6}{|c|}{ TBI patients $(n=39)$} & \multicolumn{2}{|c|}{$\mathrm{HC}(\mathrm{n}=53)$} \\
\hline & \multicolumn{2}{|c|}{ Mild $(n=4)$} & \multicolumn{2}{|c|}{ Moderate $(n=6)$} & \multicolumn{2}{|c|}{ Severe $(n=29)$} & \multirow[b]{2}{*}{ Mean } & \multirow[b]{2}{*}{ SD } \\
\hline & Mean & SD & Mean & SD & Mean & SD & & \\
\hline Gender (male/female) & \multicolumn{2}{|c|}{$2 / 2$} & \multicolumn{2}{|c|}{$2 / 4$} & \multicolumn{2}{|c|}{$19 / 10$} & \multicolumn{2}{|c|}{$26 / 27$} \\
\hline Age (years) & 44.75 & 18.77 & 49 & 17.50 & 30.97 & 13 & 35.74 & 10.94 \\
\hline NART & 108.50 & 8.85 & 113.60 & 11.52 & 108.96 & 10.54 & 112.57 & 7.40 \\
\hline ISS & 12 & 7.62 & 24.17 & 10.96 & 22.52 & 10.16 & & \\
\hline APACHE II & 10.75 & 6.13 & 17.17 & 7.73 & 16.22 & 4.08 & & \\
\hline \multirow[t]{2}{*}{ Time-post injury (months) } & 20.75 & 13.38 & 14.33 & 8.36 & 14.21 & 8.78 & & \\
\hline & Median & IQR & Median & IQR & Median & IQR & & \\
\hline GCS & 14.50 & 2 & 10.50 & 2 & 5 & 3 & & \\
\hline GOS & 4 & 1.50 & 3.5 & 2 & 4 & 1 & & \\
\hline
\end{tabular}

NART: National Adult Reading Test; GCS: Glasgow Coma Scale; GOS: Glasgow Outcome Scale; ISS: Injury Severity Scale; APACHE II: Acute Physiology and Chronic Health Evaluation II. 
Table 2. Descriptive scores for the different indices of memory functioning.

\begin{tabular}{lccccccc}
\hline & \multicolumn{2}{c}{ TBI patients $(\mathbf{n}=\mathbf{3 8})$} & \multicolumn{2}{c}{ HC $(\mathbf{n}=\mathbf{5 3})$} & \multicolumn{3}{c}{ Test } \\
\hline & Mean & SD & Mean & SD & F & $\boldsymbol{p}$ & $\boldsymbol{d}$ \\
\hline PRM & 84.32 & 14.74 & 90.72 & 9.83 & 3.939 & 0.023 & -0.51 \\
SRM & 2589.10 & 1470.70 & 1983.53 & 556.32 & 5.041 & 0.008 & 0.54 \\
PAL & 5.71 & 8.50 & 0.23 & 3.22 & 11.954 & 0.000 & 0.85 \\
\hline
\end{tabular}

PRM: Patter Recognition Memory percentage of correct trials; SRM: Spatial Recognition Memory mean latency for correct responses; PAL: Paired Associates Learning Total errors made at the 6-pattern stage. 
Table 3. Significant correlations between the measure of PRM and the volume of grey, white matter and CSF in the TBI sample.

\begin{tabular}{lcc}
\hline Location & R score & P value \\
\hline Grey matter & & \\
\hline Left middle temporal gyrus & 0.33 & 0.044 \\
Right middle occipital gyrus & 0.33 & 0.044 \\
Left angular gyrus & 0.34 & 0.040 \\
Left putamen & 0.38 & 0.021 \\
\hline White matter & & \\
\hline Right hippocampus & 0.46 & 0.004 \\
Left middle temporal gyrus & 0.34 & 0.039 \\
Right middle temporal gyrus & 0.38 & 0.020 \\
Left inferior frontal gyrus & 0.40 & 0.014 \\
Left angular gyrus & 0.40 & 0.013 \\
Left cerebellum (area 3) & 0.36 & 0.030 \\
Left cerebellum (area 10) & 0.41 & 0.013 \\
Vermis (areas 1 and 2) & 0.36 & 0.028 \\
\hline CSF & & \\
\hline Right hippocampus & -0.43 & 0.007 \\
Vermis (areas 4 and 5) & 0.37 & 0.026 \\
\hline
\end{tabular}


Table 4. Significant correlations between performance the measure of SRM and the volume of grey, white and CSF in the TBI sample.

\begin{tabular}{lcc}
\hline Location & R score & P value \\
\hline Grey matter & & \\
\hline Left hippocampus & -0.37 & 0.023 \\
Right hippocampus & -0.40 & 0.013 \\
Vermis (areas 1/2) & -0.33 & 0.045 \\
Vermis (area 3) & -0.39 & 0.017 \\
\hline White matter & & \\
\hline Left middle frontal gyrus & -0.47 & 0.003 \\
Right middle frontal gyrus & -0.34 & 0.043 \\
Left inferior frontal gyrus & -0.33 & 0.046 \\
Right inferior frontal gyrus & -0.43 & 0.007 \\
Left cuneus & 0.39 & 0.018 \\
\hline CSF & & \\
\hline Right cerebellum (area 10) & 0.35 & 0.032 \\
\hline
\end{tabular}


Table 5. Significant correlations between the measure of PAL and the volume of amount of grey,

white and CSF in the TBI sample.

\begin{tabular}{lcc}
\hline Location & R score & P value \\
\hline Grey matter & & \\
\hline Right hippocampus & -0.42 & 0.011 \\
Right inferior temporal gyrus & -0.48 & 0.003 \\
Right superior temporal gyrus & -0.33 & 0.049 \\
Right middle temporal gyrus & -0.36 & 0.031 \\
Right middle temporal pole & -0.37 & 0.026 \\
Left superior medial frontal gyrus & -0.33 & 0.047 \\
Right rolandic operculum & -0.38 & 0.022 \\
Right middle cingulum & -0.34 & 0.043 \\
Right precentral gyrus & -0.49 & 0.002 \\
Left supplementary motor area & -0.37 & 0.023 \\
Right supplementary motor area & -0.38 & 0.022 \\
Left superior parietal gyrus & -0.37 & 0.026 \\
Left angular gyrus & -0.37 & 0.024 \\
Right angular gyrus & -0.33 & 0.048 \\
Right fusiform gyrus & -0.45 & 0.005 \\
Right middle occipital gyrus & -0.39 & 0.017 \\
Right caudate nucleus & -0.38 & 0.022 \\
Right putamen & -0.37 & 0.025 \\
\hline White matter & & \\
\hline Right hippocampus & -0.55 & 0.000 \\
Right middle temporal gyrus & -0.48 & 0.003 \\
Right inferior temporal gyrus & -0.46 & 0.00 \\
Left middle temporal pole & 0.33 & 0.045 \\
Left inferior frontal gyrus & -0.34 & 0.038 \\
Right inferior frontal gyrus & -0.34 & 0.040 \\
Right superior frontal gyrus & -0.33 & 0.047 \\
Left supplementary motor area & -0.35 & 0.033 \\
Left caudate & -0.33 & 0.045 \\
Left putamen & -0.36 & 0.030 \\
Left pallidum & -0.43 & 0.009 \\
Right pallidum & -0.47 & 0.004 \\
Right thalamus & -0.37 & 0.024 \\
Left cerebellum (area 10) & -0.38 & 0.022 \\
Vermis (area 3) & -0.35 & 0.036 \\
\hline CSF & 0.41 & 0.013 \\
\hline Right hippocampus & 0.36 & 0.029 \\
Right middle temporal gyrus & 0.52 & 0.001 \\
Right inferior temporal gyrus & 0.43 & 0.009 \\
Right inferior frontal gyrus & 0.40 & 0.015 \\
Left middle occipital gyrus & 0.45 & 0.005 \\
Left superior occipital gyrus & 0.39 & 0.049 \\
\hline
\end{tabular}




\section{Figures}

Figure 1. Clusters of significant correlations found between the measure of PAL and the volume of grey, white and CSF. Cluster of correlations between the volume of grey matter of the left superior medial frontal gyrus (peak at $\mathrm{x}, \mathrm{y}, \mathrm{z}=0,54,45 ; \mathrm{t}=4.76$ ) and precuneus (peak at $\mathrm{x}, \mathrm{y}, \mathrm{z}$ $=-12,-67,61 ; \mathrm{t}=4.77$ ) and the right precentral gyrus (peak at $\mathrm{x}, \mathrm{y}, \mathrm{z}=36,-22,64 ; \mathrm{t}=5.02$ ) (negative correlation) (green), the volume of white matter near the sagittal stratum (includes inferior fronto-occipital fasciculus and inferior longitudinal fasciculus) (peak at $\mathrm{x}, \mathrm{y}, \mathrm{z}=42,-24$, 8; $\mathrm{t}=4.41$ ) (negative correlation) (red) and the CSF of the right inferior temporal (peak at $\mathrm{x}, \mathrm{y}, \mathrm{z}=$ 52, $-61,-14 ; \mathrm{t}=4.82$ ) and middle frontal gyrus (peak at $\mathrm{x}, \mathrm{y}, \mathrm{z}=44,54,12 ; \mathrm{t}=5.44$ ) and left superior parietal gyrus (peak at $\mathrm{x}, \mathrm{y}, \mathrm{z}=-16,-70,54$; $\mathrm{t}=5.17$ ) (positive correlation) (blue). Results are overlaid on axial and sagittal sections of a normalized brain.

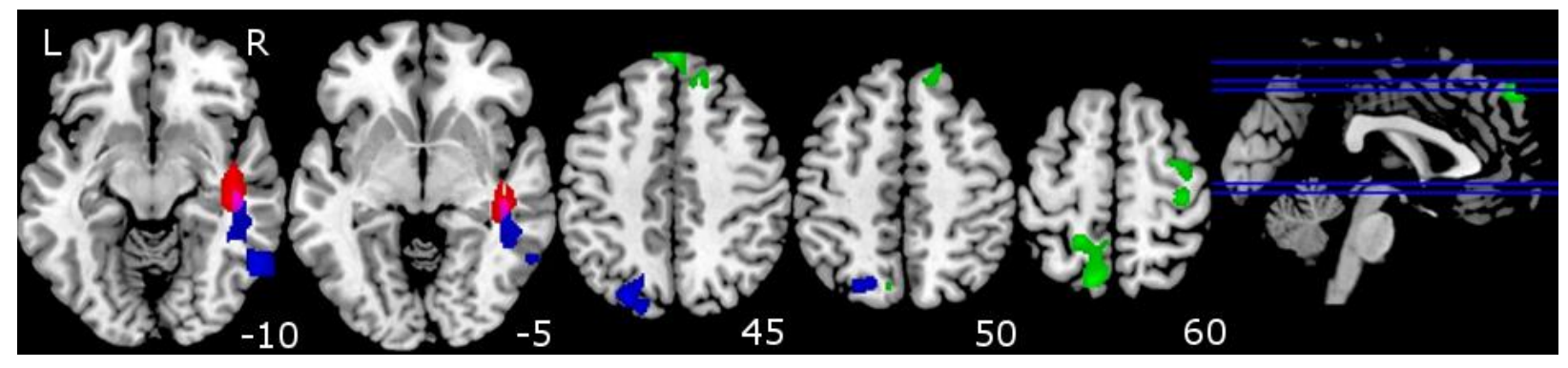




\section{Supplementary Material}

Figure S1. Illustration of analyses of different levels of resolution, including whole brain-, hemisphere-, lobe-, gyral- and voxel-level.

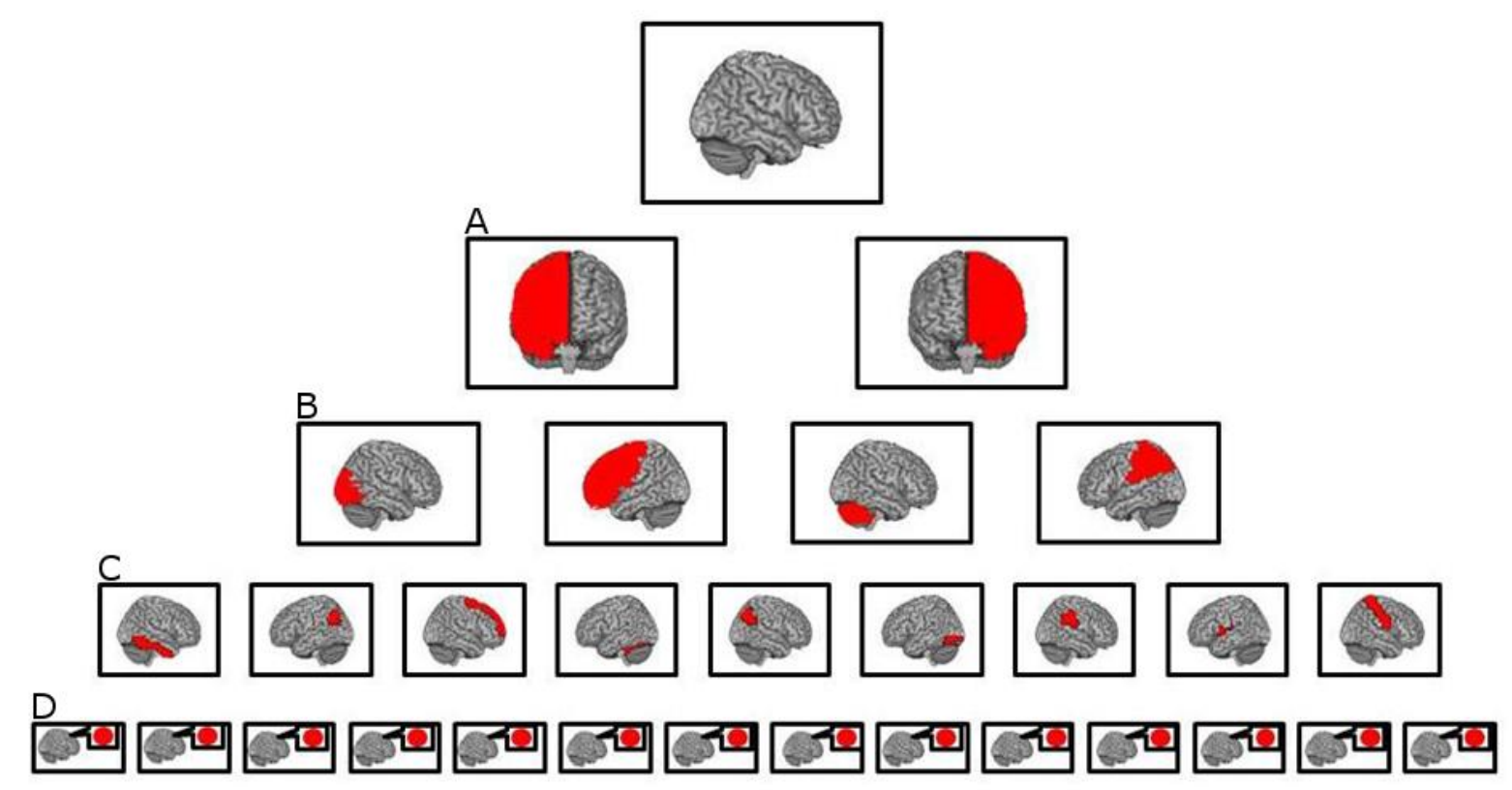

